\title{
Generalized Fibonacci Polynomials
}

\author{
Yashwant K. Panwar ${ }^{1, *}$, B. Singh ${ }^{2}$, V.K. Gupta ${ }^{3}$ \\ ${ }^{1}$ Mandsaur Institute of Technology, Mandsaur, India \\ ${ }^{2}$ School of Studies in Mathematics, Vikram University, Ujjain, India \\ ${ }^{3}$ Govt. Madhav Science College, Ujjain, India \\ *Corresponding author: yashwantpanwar@gmail.com
}

Received August 22, 2013; Revised October 24, 2013; Accepted November 10, 2013

\begin{abstract}
In this study, we present generalized Fibonacci polynomials. We have used their Binet's formula and generating function to derive the identities. The proofs of the main theorems are based on special functions, simple algebra and give several interesting properties involving them.
\end{abstract}

Keywords: generalized Fibonacci polynomials, Binet's formula, generating function

Cite This Article: Yashwant K. Panwar, B. Singh, and V.K. Gupta, "Generalized Fibonacci Polynomials." Turkish Journal of Analysis and Number Theory 1, no. 1 (2013): 43-47. doi: 10.12691/tjant-1-1-9.

\section{Introduction}

Fibonacci polynomials are a great importance in mathematics. Large classes of polynomials can be defined by Fibonacci-like recurrence relation and yield Fibonacci numbers [15]. Such polynomials, called the Fibonacci polynomials, were studied in 1883 by the Belgian Mathematician Eugene Charles Catalan and the German Mathematician E. Jacobsthal.

The polynomials $f_{n}(x)$ studied by Catalan are defined by the recurrence relation

$$
f_{n+2}(x)=x f_{n+1}(x)+f_{n}(x)
$$

where $f_{1}(x)=1, f_{2}(x)=x$, and $n \geq 3$. Notice that $f_{n}(1)=F$, the nth Fibonacci number.

The Fibonacci polynomials studied by Jacobsthal were defined by

$$
J_{n}(x)=J_{n-1}(x)+x J_{n-2}(x)
$$

where $J_{1}(x)=1=J_{2}(x)$, and $n \geq 3$.

The Pell polynomials $p_{n}(x)$ are defined by

$$
p_{n}(x)=2 x p_{n-1}(x)+p_{n-2}(x)
$$

where $p_{0}(x)=0, p_{1}(x)=1$, and $n \geq 2$.

The Lucas polynomials ( ) $n l x$, originally studied in 1970 by Bicknell, are defined by

$$
l_{n}(x)=x l_{n-1}(x)+l_{n-2}(x)
$$

where $l_{0}(x)=2, l_{1}(x)=x$, and $n \geq 2$.

It is well known that the Fibonacci polynomials and Lucas polynomials are closely related. Obviously, they have a deep relationship with the famous Fibonacci and
Lucas sequences. That is $f_{n}(1)=F_{n}$ and $l_{n}(1)=L_{n}$, where $F_{n}$ and $L_{n}$ are the Fibonacci and Lucas numbers. Swamy [11] defined the Fibonacci Polynomials and obtained some more identities for these polynomials. Hogget and Lind [17] make a similar "symbolic substitution" of certain sequences into the Fibonacci polynomials, they extend these results to the substitution of any recur rent sequence into any sequence of polynomials obeying a recurrence relation with polynomial coefficients. Since then many problems about the polynomials have been proposed in various issue of the Fibonacci Quarterly. Hoggatt, Philips and Leonard [16] have obtained some more identities involving Fibonacci Polynomials and Lucas polynomials. A. Lupas [3] present many interesting properties of Fibonacci and Lucas Polynomials. C. Berg [4] defined Fibonacci numbers and orthogonal polynomials. S. Falcon and A. Plaza [13] defined the k-Fibonacci polynomials are the natural extension of the k-Fibonacci numbers and many of their properties admit a straightforward proof and many relations for the derivatives of Fibonacci polynomials are proven. K. Kaygisiz and A. Sahin [10] present new generalizations of the Lucas numbers by matrix representation, using Generalized Lucas Polynomials. G. Y. Lee and M. Asci [8], consider the Pascal matrix and define a new generalization of Fibonacci polynomials called (p, q)-Fibonacci polynomials. They obtain combinatorial identities and by using Riordan method they get a factorizations of Pascal matrix involving (p, q)Fibonacci polynomials. Many authors have studied Fibonacci polynomials. In this paper, we present generalization of Fibonacci and Lucas Polynomials by changing the initial terms but the recurrence relation is preserved.

\section{Generalized Fibonacci Polynomials}

The generalized Fibonacci polynomials defined by 


$$
f_{n+1}(x)=\left\{\begin{array}{cc}
s & \text { if } n=0 \\
s x & \text { if } n=1 \\
x f_{n}(x)+f_{n-1}(x) & \text { if } n \geq 2
\end{array}\right\}
$$

If $s=1$, then we obtained classical Fibonacci polynomial sequence.

It is well known that the Fibonacci polynomials and Lucas Polynomials are closely related. The

generalized Lucas polynomials defined by

$$
l_{n}(x)=\left\{\begin{array}{cc}
2 s & \text { if } n=0 \\
s x & \text { if } n=1 \\
x l_{n-1}(x)+l_{n-2}(x) & \text { if } n \geq 2
\end{array}\right\}
$$

If $s=1$, then we obtained classical Lucas polynomial sequence.

In the 19th century, the French mathematician Binet devised two remarkable analytical formulas for the Fibonacci and Lucas numbers. In our case, Binet's formula allows us to express the generalized Fibonacci Polynomials in function of the roots $\Re_{1} \& \Re_{2}$ of the following characteristic equation, associated to the recurrence relation $(2.1) \&(2.2)$ :

$$
x^{2}=x t+1
$$

\section{Properties of Generalized Fibonacci Polynomials}

Theorem 1: (Binet's formula). The nth generalized Fibonacci Polynomials is given by

$$
f_{n}(x)=s \frac{\mathfrak{R}_{1}^{n}-\mathfrak{R}_{2}^{n}}{\mathfrak{R}_{1}-\mathfrak{R}_{2}}
$$

where are the roots of the characteristic equation (3), $\mathfrak{R}_{1}>\mathfrak{R}_{2}$ and $\mathfrak{R}_{1}=\frac{x+\sqrt{x^{2}+4}}{2}$ and $\mathfrak{R}_{2}=\frac{x-\sqrt{x^{2}+4}}{2}$.

Proof: we use the Principle of Mathematical Induction (PMI) on $\mathrm{n}$. It is clear the result is true for $\mathrm{n}=0$ and $\mathrm{n}=1$ by hypothesis. Assume that it is true for $i$ such that $0 \leq i \leq$ $\mathrm{r}+1$, then

$$
f_{i}(x)=s \frac{\mathfrak{R}_{1}^{i}-\mathfrak{R}_{2}^{i}}{\mathfrak{R}_{1}-\mathfrak{R}_{2}}
$$

It follows from definition of generalized Fibonacci Polynomials and from equation (3.1),

$$
f_{r+2}(x)=x f_{t+1}(x)+f_{r}(x)=s \frac{\Re_{1}^{r+2}-\mathfrak{R}_{2}^{r+2}}{\mathfrak{R}_{1}-\mathfrak{R}_{2}}
$$

Thus, the formula is true for any positive integer $\mathrm{n}$.

Theorem 2: (Binet's formula). The nth generalized Lucas Polynomials is given by

$$
l_{n}(x)=s\left(\mathfrak{R}_{1}^{n}+\mathfrak{R}_{2}^{n}\right)
$$

Proposition 3: For any integer $\mathrm{n} \geq 1$,

$$
\begin{aligned}
& \mathfrak{R}_{1}^{n+2}=x \Re_{1}^{n+1}+\Re_{1}^{n} \\
& \Re_{2}^{n+2}=x \Re_{2}^{n+1}+\Re_{2}^{n}
\end{aligned}
$$

Proof: Since $\mathfrak{R}_{1} \& \Re_{2}$ are the roots of the characteristic equation (2.3), then

$$
\begin{aligned}
& \mathfrak{R}_{1}^{1}=x \mathfrak{R}_{1}+1 \\
& \mathfrak{R}_{2}^{2}=x \Re_{2}+2
\end{aligned}
$$

now, multiplying both sides of these equations by $\mathfrak{R}_{1}^{n} \& \mathfrak{R}_{2}^{n}$ respectively, we obtain the desired result.

Proposition 4: For any integer $n \geq 1$,

$$
s\left(\mathfrak{R}_{1}^{n}+\mathfrak{R}_{2}^{n}\right)=f_{n+1}(x)+f_{n-1}(x)
$$

Proof: By using Eq. (3.1) in the R.H.S. of Eq. (3.5) and taking in to account that $\Re_{1}=\frac{-1}{\mathfrak{R}_{2}}$ it is obtained

$$
\begin{aligned}
(R H S) & =s \frac{\mathfrak{R}_{1}^{n+1}-\mathfrak{R}_{2}^{n+1}}{\mathfrak{R}_{1}-\mathfrak{R}_{2}}+s \frac{\mathfrak{R}_{1}^{n-1}-\mathfrak{R}_{2}^{n-1}}{\mathfrak{R}_{1}-\mathfrak{R}_{2}} \\
& =\frac{s}{\mathfrak{R}_{1}-\mathfrak{R}_{2}}\left(\mathfrak{R}_{1}^{n+1}+\mathfrak{R}_{1}^{n-1}-\mathfrak{R}_{2}^{n+1}-\mathfrak{R}_{2}^{n-1}\right) \\
& =\frac{s}{\mathfrak{R}_{1}-\mathfrak{R}_{2}}\left\{\mathfrak{R}_{1}^{n}\left(\mathfrak{R}_{1}+\frac{1}{\mathfrak{R}_{1}}\right)-\mathfrak{R}_{2}^{n}\left(\mathfrak{R}_{2}+\frac{1}{\mathfrak{R}_{2}}\right)\right\} \\
& =s\left(\mathfrak{R}_{1}^{n}+\mathfrak{R}_{2}^{n}\right)
\end{aligned}
$$

Proposition 5: For any integer $n$,

$$
\left(x^{2}+4\right) f_{n}^{2}(x)+4 s^{2}(-1)^{n}=\left\{\begin{array}{l}
s^{2}\left(\mathfrak{R}_{1}^{n}+\mathfrak{R}_{2}^{n}\right)^{2} ; \text { if } n \text { is even } \\
s^{2}\left(\mathfrak{R}_{1}^{n}-\mathfrak{R}_{2}^{n}\right)^{2} ; \text { if } n \text { is odd }
\end{array}\right.
$$

Proof: From the Binet's formula of generalized Fibonacci Polynomials

$$
f_{n}^{2}(x)=\frac{s^{2}}{\left(\Re_{1}-\mathfrak{R}_{2}\right)^{2}}\left\{\mathfrak{R}_{1}^{2 n}-2\left(\mathfrak{R}_{1} \Re_{2}\right)^{n}+\mathfrak{R}_{2}^{2 n}\right\}
$$

If $\mathrm{n}$ is even, $\left(x^{2}+4\right) f_{n}^{2}(x)+4 s^{2}=\left(s \Re_{1}^{n}+s \Re_{2}^{n}\right)^{2}$

If $\mathrm{n}$ is odd, $\left(x^{2}+4\right) f_{n}^{2}(x)-4 s^{2}=\left(s \Re_{1}^{n}-s \Re_{2}^{n}\right)^{2}$

Let us denote $\left(s \Re_{1}^{n}+s \Re_{2}^{n}\right)$ by $l_{n}(x)$.

Then previous formula become:

$$
\left(x^{2}+4\right) f_{n}^{2}(x)+4 s^{2}(-1)^{n}=l_{n}^{2}(x)
$$

\section{Sums of Generalized Fibonacci Polynomials}

In this section, we study the sums of generalized Fibonacci Polynomials. This enables us to give in a straightforward way several formulas for the sums of such Polynomials.

Lemma 6: For fixed integers $p, q$ with $0 \leq q \leq p-1$, the following equality holds

$$
s f_{p(n+2)}(x)=l_{p}(x) f_{p(n+1)+q}(x)-(-1)^{p} s f_{p n+q}(x) \text { (3.8) }
$$


Proof: From the Binet's formula of generalized Fibonacci and Lucas Polynomials,

$$
\begin{aligned}
& l_{p}(x) f_{p(n+1)+q}(x) \\
& =s\left(\mathfrak{R}_{1}^{n}+\mathfrak{R}_{2}^{n}\right) s\left[\frac{\mathfrak{R}_{1}^{p(n+1)+q}-\mathfrak{R}_{2}^{p(n+1)+q}}{\mathfrak{R}_{1}-\mathfrak{R}_{2}}\right] \\
& =\frac{s^{2}}{\mathfrak{R}_{1}-\mathfrak{R}_{2}}\left[\mathfrak{R}_{1}^{p(n+2)+q}+(-1)^{p} \mathfrak{R}_{1}^{p n+q}-(-1)^{p} \mathfrak{R}_{2}^{p n+q}-\mathfrak{R}_{2}^{p(n+2)+q}\right] \\
& =\frac{s^{2}}{\mathfrak{R}_{1}-\mathfrak{R}_{2}}\left\{\left[\mathfrak{R}_{1}^{p(n+2)+q}-\mathfrak{R}_{2}^{p(n+2)+q}\right]+(-1)^{p}\left(\mathfrak{R}_{1}^{p n+q}-\mathfrak{R}_{2}^{p n+q}\right)\right\} \\
& =s f_{p(n+2)}(x)+(-1)^{p} s f_{p n+q}(x)
\end{aligned}
$$

then, the equation becomes,

$$
s f_{p(n+2)}(x)=l_{p}(x) f_{p(n+1)+q}(x)-(-1)^{p} s f_{p n+q}(x)
$$

Proposition 7: For fixed integers $p, q$ with $0 \leq q \leq p-1$, the following equality holds

$$
\sum_{i=0}^{n} f_{p i+q}(x)=\frac{f_{p(n+1)+q}(x)-f_{q}(x)-(-1)^{p} f_{p n+q}(x)-(-1)^{p} f_{p-q}(x)}{l_{p}(x)-(-1)^{p}-1}
$$

Proof: Applying Binet's formula of generalized Fibonacci Polynomials,

$$
\begin{aligned}
& \sum_{i=0}^{n} f_{p i+q}(x) \\
& =s \sum_{i=0}^{n} \frac{\Re_{1}^{p i+q}-\mathfrak{R}_{2}^{p i+q}}{\mathfrak{R}_{1}-\mathfrak{R}_{2}} \\
& =\frac{s}{\Re_{1}-\mathfrak{R}_{2}}\left(\sum_{i=0}^{n} \mathfrak{R}_{1}^{p i+q}-\sum_{i=0}^{n} \mathfrak{R}_{2}^{p i+q}\right) \\
& =\frac{s}{\mathfrak{R}_{1}-\mathfrak{R}_{2}}\left(\frac{\mathfrak{R}_{1}^{p n+q+p}-\mathfrak{R}_{1}^{q}}{\mathfrak{R}_{1}^{p}-1}-\frac{\mathfrak{R}_{2}^{p n+q+p}-\mathfrak{R}_{2}^{q}}{\mathfrak{R}_{2}^{p}-1}\right) \\
& =\frac{s}{(-1)^{p}-l_{p}(x)+1}\left[(-1)^{p} f_{p n+q}(x)-f_{p(n+1)+q}(x)+f_{q}(x)+(-1)^{q} f_{p-q}(x)\right] \\
& =\frac{f_{p(n+1)+q}(x)-f_{q}(x)-(-1)^{p} f_{p n+q}(x)-(-1)^{q} f_{p-q}(x)}{l_{p}(x)-(-1)^{p}-1}
\end{aligned}
$$

Corollary 7.1: Sum of odd generalized Fibonacci polynomials

If $p=2 m+1$, then Eq.(3.9) is

$$
\sum_{i=0}^{n} f_{(2 m+1) i+q}(x)=\frac{f_{(2 m+1)(n+1)+q}(x)+f_{(2 m+1) n+q}(x)-f_{q}(x)-(-1)^{q} f_{(2 m+1)-q}(x)}{l_{2 m+1}(x)}
$$

For example

(1) If $m=0$ then $p=1$

$$
\sum_{i=0}^{n} f_{i+q}(x)=\frac{f_{n+q+1}(x)+f_{n+q}(x)-f_{q}(x)-(-1)^{q} f_{1-q}(x)}{s x}
$$

(i) For $q=0$ : $\sum_{i=0}^{n} f_{i}(x)=\frac{f_{n+1}(x)+f_{n}(x)-s}{s x}$

(2) If $m=0$ then $p=3$

$$
\sum_{i=0}^{n} f_{3 i+q}(x)=\frac{f_{3 n+q+3}(x)+f_{3 n+q}(x)-f_{q}(x)-(-1)^{q} f_{3-q}(x)}{s x^{3}+3 s x}(3.12)
$$

(i) For $q=0$ : $\sum_{i=0}^{n} f_{3 i}(x)=\frac{f_{3 n+3}(x)+f_{3 n}(x)-f_{3-q}(x)}{s x^{2}+s}$

(ii) For $q=1$ : $\sum_{i=0}^{n} f_{3 i+1}(x)=\frac{f_{3 n+4}(x)+f_{3 n+1}(x)-s+s x}{s x^{2}+s}$ (iii) For $q=2$ : $\sum_{i=0}^{n} f_{3 i+2}(x)=\frac{f_{3 n+5}(x)+f_{3 n+2}(x)-s-s x}{s x^{2}+s}$

(2) If $m=2$ then $p=5$

$$
\sum_{i=0}^{n} f_{5 i+q}(x)=\frac{f_{5 n+q+5}(x)+f_{5 n+q}(x)-f_{q}(x)-(-1)^{q} f_{5-q}(x)}{s x^{5}+5 s x^{3}+5 s x}
$$

(i) For $q=0: \sum_{i=0}^{n} f_{5 i}(x)=\frac{f_{5 n+5}(x)+f_{5 n}(x)-\left(s x^{4}+3 s x^{2}+s\right)}{s x^{5}+5 s x^{3}+5 s x}$

(ii) For $q=1: \sum_{i=0}^{n} f_{5 i+1}(x)=\frac{f_{5 n+6}(x)+f_{5 n+1}(x)+s x^{3}+2 s x-s}{s x^{5}+5 s x^{3}+5 s x}$

(iii) For $q=2: \sum_{i=0}^{n} f_{5 i+2}(x)=\frac{f_{5 n+7}(x)+f_{5 n+2}(x)-\left(s x^{2}+s x+s\right)}{s x^{5}+5 s x^{3}+5 s x}$

(iv) For $q=3$ : $\sum_{i=0}^{n} f_{5 i+3}(x)=\frac{f_{5 n+8}(x)+f_{5 n+3}(x)-\left(s x^{2}-s x+s\right)}{s x^{5}+5 s x^{3}+5 s x}$

(v) For $q=4: \sum_{i=0}^{n} f_{5 i+4}(x)=\frac{f_{5 n+9}(x)+f_{5 n+4}(x)-\left(s x^{3}+2 s x+s\right)}{s x^{5}+5 s x^{3}+5 s x}$

Corollary 7.2: Sum of even generalized Fibonacci polynomials

If $p=2 m$, then Eq.(3.9) is

$\sum_{i=0}^{n} f_{2 m i+q}(x)=\frac{f_{2 m(n+1)+q}(x)+f_{2 m n+q}(x)-f_{q}(x)-(-1)^{q} f_{2 m-q}(x)}{l_{2 m}(x)-2}(3.14)$

For example

(1) If $m=1$ then $p=2$

$\sum_{i=0}^{n} f_{2 i+q}(x)=\frac{f_{2 n+2+q}(x)+f_{2 n+q}(x)-f_{q}(x)-(-1)^{q} f_{2-q}(x)}{s x^{2}+2 s-2}(3.15)$

(i) For $q=0: \sum_{i=0}^{n} f_{2 i}(x)=\frac{f_{2 n+2}(x)-f_{2 n}(x)-s x}{s x^{2}+2 s-2}$

(ii) For $q=1: \sum_{i=0}^{n} f_{2 i+1}(x)=\frac{f_{2 n+3}(x)-f_{2 n+1}(x)-s x}{s x^{2}+2 s-2}$

(iii) For $q=2$ : $\sum_{i=0}^{n} f_{2 i+2}(x)=\frac{f_{2 n+4}(x)-f_{2 n+2}(x)-s x}{s x^{2}+2 s-2}$

(2) If $m=2$ then $p=4$

$\sum_{i=0}^{n} f_{4 i+q}(x)=\frac{f_{4 n+4+q}(x)+f_{4 n+q}(x)-f_{q}(x)-(-1)^{q} f_{4-q}(x)}{s x^{4}+4 s x^{2}+2 s-2}(3.16)$

(i) For $q=0$ : $\sum_{i=0}^{n} f_{4 i}(x)=\frac{f_{4 n+4}(x)-f_{4 n}(x)-s x^{3}-2 s x}{s x^{4}+4 s x^{2}+2 s-2}$

(ii) For $q=1: \sum_{i=0}^{n} f_{4 i+1}(x)=\frac{f_{4 n+5}(x)-f_{4 n+1}(x)+s x^{2}}{s x^{4}+4 s x^{2}+2 s-2}$

(iii) For $q=2$ : $\sum_{i=0}^{n} f_{4 i+2}(x)=\frac{f_{4 n+6}(x)-f_{4 n+2}(x)-s x}{s x^{4}+4 s x^{2}+2 s-2}$

(iv) For $q=3$ : $\sum_{i=0}^{n} f_{4 i+3}(x)=\frac{f_{4 n+7}(x)-f_{4 n+3}(x)-s x^{2}}{s x^{4}+4 s x^{2}+2 s-2}$

(v) For $q=4$ : $\sum_{i=0}^{n} f_{4 i+4}(x)=\frac{f_{4 n+8}(x)-f_{4 n+4}(x)-s x^{3}-2 s x}{s x^{4}+4 s x^{2}+2 s-2}$

(3) If $m=3$ then $p=6$

$\sum_{i=0}^{n} f_{6 i+q}(x)=\frac{f_{6 n+6+q}(x)-f_{6 n+q}(x)-f_{q}(x)-(-1)^{q} f_{6-q}(x)}{s x^{6}+6 s x^{4}+9 s x^{2}+2 s-2}(3.17)$ 
(i) For $q=0$ : $\sum_{i=0}^{n} f_{6 i}(x)=\frac{f_{6 n+6}(x)-f_{6 n}(x)-s x^{6}-6 s x^{4}-9 s x^{2}-2 s}{s x^{6}+6 s x^{4}+9 s x^{2}+2 s-2}$

(ii) For $q=1: \sum_{i=0}^{n} f_{6 i+1}(x)=\frac{f_{6 n+7}(x)-f_{6 n+1}(x)+s x^{4}+3 s x^{2}}{s x^{6}+6 s x^{4}+9 s x^{2}+2 s-2}$

(iii) For $q=2$ : $\sum_{i=0}^{n} f_{6 i+2}(x)=\frac{f_{6 n+8}(x)-f_{6 n+2}(x)+s x^{4}+3 s x^{2}}{s x^{6}+6 s x^{4}+9 s x^{2}+2 s-2}$

(iv) For $q=3$ : $\sum_{i=0}^{n} f_{6 i+3}(x)=\frac{f_{6 n+9}(x)-f_{6 n+3}(x)}{s x^{6}+6 s x^{4}+9 s x^{2}+2 s-2}$

Proposition 8: For fixed integers $p, q$ with $0 \leq q \leq p-1$, the following equality holds

$$
\begin{gathered}
\sum_{i=0}^{n}(-1)^{i} f_{p i+q}(x)= \\
\frac{(-1)^{p} f_{p(n+1)+q}(x)+(-1)^{n+p} f_{p n+q}(x)+(-1)^{q+1} f_{p-q}(x)+f_{q}(x)}{l_{p}(x)+(-1)^{p}+1}
\end{gathered}
$$

Proof: Applying Binet's formula of generalized Fibonacci Polynomials, the proof is clear. For different values of $p \& q$ :
(i) $\sum_{i=0}^{n}(-1)^{i} f_{i}(x)=\frac{(-1)^{n} f_{n+1}(x)-(-1)^{n} f_{n}(x)-s}{s x}$
(ii) $\sum_{i=0}^{n}(-1)^{i} f_{2 i}(x)=\frac{(-1)^{n} f_{2 n+2}(x)+(-1)^{n} f_{2 n}(x)-s x}{s x^{2}+2 s+2}$
(iii) $\sum_{i=0}^{n}(-1)^{i} f_{2 i+1}(x)=\frac{(-1)^{n} f_{2 n+3}(x)+(-1)^{n} f_{2 n+1}(x)-s x}{s x^{2}+2 s+2}$
(iv) $\sum_{i=0}^{n}(-1)^{i} f_{4 i}(x)=\frac{(-1)^{n} f_{4 n+4}(x)+(-1)^{n} f_{4 n}(x)-s x^{3}-2 s x}{s x^{4}+4 s x^{2}+2 s+2}$
(v) $\sum_{i=0}^{n}(-1)^{i} f_{4 i+1}(x)=\frac{(-1)^{n} f_{4 n+5}(x)+(-1)^{n} f_{4 n+1}(x)+s x^{2}+2 s}{s x^{4}+4 s x^{2}+2 s+2}$
(vi) $\sum_{i=0}^{n}(-1)^{i} f_{4 i+2}(x)=\frac{(-1)^{n} f_{4 n+6}(x)+(-1)^{n} f_{4 n+2}(x)}{s x^{4}+4 s x^{2}+2 s+2}$
(vii) $\sum_{i=0}^{n}(-1)^{i} f_{4 i+3}(x)=\frac{(-1)^{n} f_{4 n+7}(x)+(-1)^{n} f_{4 n+3}(x)+s x^{2}+2 s}{s x^{4}+4 s x^{2}+2 s+2}$

\section{Confluent Hypergeometric Identities of Generalized Fibonacci Polynomials}

A. Lupas [3], present a guide of Fibonacci and Lucas Polynomial and defined Fibonacci and Lucas Polynomial in terms of hypergeometric form. K. Dilcher [9], defined Fibonacci numbers in terms of hypergeometric function. C. Berg [4], defined Fibonacci numbers and orthogonal polynomials.

In this section, we established some properties of generalized Fibonacci Polynomials in terms of confluent hypergeometric function. Proofs of the theorem are based on special function, simple algebra and give several interesting identities involving them.

Theorem 9: If $f_{n}(x)$ and $l_{n}(x)$ are generalized Fibonacci and Lucas Polynomials then

$$
\sum_{n=0}^{\infty} f_{n}(x) \frac{t^{n-1}}{n !}=s e^{x t}{ }_{1} F_{o}\left(n+1,-, t^{2}\right)
$$

(ii) $\sum_{n=0}^{\infty} l_{n}(x) \frac{t^{n}}{n !}=\delta s e^{x t}{ }_{1} F_{o}\left(n+1,-, t^{2}\right)$, where $\delta=2-x t$

$$
\begin{gathered}
l_{n}(x)=2 f_{n+1}(x)-x f_{n}(x) \\
l_{n+1}(x)=x f_{n+1}(x)+2 x f_{n}(x)
\end{gathered}
$$

Proof (i): Since the generating function of the generalized Fibonacci Polynomials is,

$$
\begin{aligned}
\sum_{n=0}^{\infty} f_{n}(x) t^{n-1}= & s\left(1-x t-t^{2}\right)^{-1} \\
& =s \sum_{n=0}^{\infty} t^{n}(x+t)^{n} \\
& =s \sum_{n=0}^{\infty} t^{n} \sum_{r=0}^{n} t^{r} x^{n-r}{ }^{n} C_{r} \\
& =s \sum_{n=0}^{\infty} \sum_{r=0}^{n} \frac{t^{n+r} x^{n-r} n !}{r !(n-r) !} \\
& =s \sum_{n=0}^{\infty} \sum_{r=0}^{\infty} \frac{t^{n+2 r} x^{n}(n+r) !}{r ! n !} \\
& =s \sum_{n=0}^{\infty} \frac{(x t)^{n}}{n !} \sum_{r=0}^{\infty} \frac{t^{2 r}}{r !} n !(n+1)_{r} \\
\sum_{n=0}^{\infty} f_{n}(x) \frac{t^{n-1}}{n !} & =s e^{x t}{ }_{1} F_{0}\left(n+1,-, t^{2}\right)
\end{aligned}
$$

Proof (ii): Since the generating function of the generalized Lucas Polynomials is,

$$
\begin{aligned}
& \sum_{n=0}^{\infty} l_{n}(x) t^{n}=\delta s\left(1-x t-t^{2}\right)^{-1} \\
&=\delta s \sum_{n=0}^{\infty} t^{n}(x+t)^{n} \\
&=\delta s \sum_{n=0}^{\infty} t^{n} \sum_{r=0}^{n} t^{r} x^{n-r}{ }^{n} C_{r} \\
&=\delta s \sum_{n=0}^{\infty} \sum_{r=0}^{n} \frac{t^{n+r} x^{n-r} n !}{r !(n-r) !} \\
&=\delta s \sum_{n=0}^{\infty} \sum_{r=0}^{\infty} \frac{t^{n+2 r} x^{n}(n+r) !}{r ! n !} \\
&=\delta s \sum_{n=0}^{\infty} \frac{(x t)^{n}}{n !} \sum_{r=0}^{\infty} \frac{t^{2 r}}{r !} n !(n+1)_{r} \\
& \sum_{n=0}^{\infty} l_{n}(x) \frac{t^{n}}{n !}=\delta s e^{x t}{ }_{1} F_{0}\left(n+1,-, t^{2}\right)
\end{aligned}
$$

We can easily get the following recurrence relation by using (4.1) and (4.2)

$$
\begin{aligned}
& \text { (iii) } l_{n}(x)=2 f_{n+1}(x)-x f_{n}(x) \\
& \text { (iv) } l_{n+1}(x)=x f_{n+1}(x)+2 x f_{n}(x)
\end{aligned}
$$

Theorem 10: If $f_{n}(x)$ and $l_{n}(x)$ are generalized Fibonacci and Lucas Polynomials then 
(i)

$$
\begin{gathered}
(n+1) \int \frac{f_{n+1}(x)}{l_{n+1}(x)} d x=\log c\left\{\frac{l_{n+1}(x)}{s}\right\} \\
\int f_{n+1}(x) e^{l_{n+1}(x)} d x=\frac{e^{l_{n+1}(x)}}{n+1}+c
\end{gathered}
$$

(ii)

(iii)

$$
\int_{-1}^{1} f_{n+1}(x) l_{n+1}(x) d x=0
$$

Generating functions are very helpful in finding of relations for sequences of integers. Some authors found miscellaneous identities for the Fibonacci polynomials and Lucas polynomials by manipulation with their generating functions. Our approach is rather different in this section.

Corollary 10.1:

$$
\sum_{n=0}^{\infty}\left\{f_{n}(x)+f_{n+1}(x)\right\} t^{n}=\frac{S(1+t)}{t\left(1-x t-t^{2}\right)}
$$

\section{Corollary 10.3:}

$$
\sum_{n=0}^{\infty}\left\{l_{n}(x)+l_{n+1}(x)\right\} t^{n}=\frac{2 s(1-x t+t)}{t\left(1-x t-t^{2}\right)}
$$

Proposition 11: Prove that

$$
\sum_{n=0}^{\infty}(1+n) t^{n} f_{n}(x)=\frac{s t(2-x t)}{\left(1-x t-t^{2}\right)^{2}}
$$

Proof: Using the generating function, the proof is clear.

\section{Conclusion}

We have derived many fundamental properties in this paper. We describe sums of generalized Fibonacci Polynomials. This enables us to give in a straightforward way several formulas for the sums of such Polynomials. These identities can be used to develop new identities of polynomials. Also we describe some confluent hypergeometric identities of generalized Fibonacci and Lucas polynomials. In Theorem: 10 we use $\mathbf{c}$, is the arbitrary constants of integration and give several interesting identities involving them.

\section{References}

[1] A. F. Horadam, "Extension of a synthesis for a class of polynomial sequences," The Fibonacci Quarterly, vol. 34; 1966, no. 1, 68-74.

[2] Nalli and P. Haukkanen, "On generalized Fibonacci and Lucas polynomials," Chaos, Solitons and Fractals, vol. 42; 2009, no. 5, 3179-3186

[3] Alexandru Lupas, A Guide of Fibonacci and Lucas Polynomial, Octagon Mathematics Magazine, vol. 7(1); 1999, 2-12.

[4] Christian Berg, Fibonacci numbers and orthogonal polynomials, Arab Journal of Mathematical Sciences, vol.17; 2011, 75-88.

[5] E. Artin, Collected Papers, Ed. S. Lang and J. T. Tate, New York, springer-Vaerlag, 1965.

[6] E. D. Rainville, Special Function, Macmillan, New York, 1960.

[7] G. S. Cheon, H. Kim, and L. W. Shapiro, "A generalization of Lucas polynomial sequence," Discrete Applied Mathematics, vol. $157 ; 2009$, no. $5,920-927$

[8] G. Y. Lee and M. Asci, Some Properties of the (p, q)-Fibonacci and $(p, q)$-Lucas Polynomials, Journal of Applied Mathematics, Vol. 2012, Article ID 264842, 18 pages, 2012.

[9] Karl Dilcher, "Hypergeometric functions and Fibonacci numbers", The Fibonacci Quarterly, vol. 38; 2000, no. 4, 342-363.

[10] K. Kaygisiz and A. Sahin, New Generalizations of Lucas Numbers, Gen. Math. Notes, Vol. 10; 2012, no. 1, 63-77.

[11] M. N. S. Swamy, "Problem B - 74", The Fibonacci Quarterly, vol. $3 ; 1965$, no. 3, 236.

[12] N. Robbins, Vieta's triangular array and a related family of polynomials, Internat. J. Mayth. \& Math. Sci., Vol. 14; 1991, no. 2 239-244.

[13] S. Falcon and A. Plaza, On k-Fibonacci sequences and polynomials and their derivatives, Chaos, Solitons and Fractals 39; 2009. 1005-1019.

[14] S. Vajda, Fibonacci \& Lucas numbers, and the Golden Section. Theory and applications, Chichester: Ellis Horwood, 1989.

[15] T. Koshy, Fibonacci and Lucas Numbers with Applications, Toronto, New York, NY, USA, 2001.

[16] V. E. Hoggatt, Jr., Leonard, H. T. Jr. and Philips, J. W., Twenty four Master Identities, The Fibonacci Quarterly, Vol. 9; 1971, no. $1,1-17$.

[17] V. E. Hoggatt, Jr. and D. A. Lind, Symbolic Substitutions in to Fibonacci Polynomials, The Fibonacci Quarterly, Vol. 6; 1968, no. $5,55-74$. 